\title{
Fatores associados à autopercepção negativa de saúde em mulheres climatéricas
}

\author{
Factors associated with negative self-rated health \\ in menopausal women
}

Vitor Hipólito Silva ${ }^{1}$ Josiane Santos Brant Rocha ${ }^{1}$ Antonio Prates Caldeira ${ }^{2}$

${ }^{1}$ Faculdades Integradas Pitágoras. Av. Profa. Aida Mainartina Paraiso 80, Ibituruna. 39408-007 Montes Claros MG Brasil. vitorhipolito@bol.com.br ${ }^{2}$ Departamento de Saúde da Mulher e da Criança, Universidade Estadual de Montes Claros. Montes Claros MG Brasil.

\begin{abstract}
The scope of this study was to investigate the prevalence and factors associated with negative self-rated health in menopausal women registered with the Family Health Strategy in a Brazilian urban center. It is a cross-sectional study with a random sample of menopausal women. A validated instrument addressing socio-demographic and behavioral data related to self-rated health status was used. The association between the variables studied and negative self-rated health was assessed by bivariate analysis followed by Poisson regression with robust variance, in a hierarchical model. The prevalence of negative self-rated health among the population studied was $41.6 \%$, among 761 women. Among women aged 52-65 years old, $49.2 \%$ had negative self-rated health. Age corresponding to post-menopause, education up to eight years of study, having a partner, not having a formal job, current tobacco use and physical inactivity were associated with negative self-rated health. The presence of menopausal symptoms, overweight and obesity, the current use of medication and the presence of chronic diseases were also associated with negative self-rated health in the final mod$e l$. The associations observed point to the need for health promotion activities aimed at menopausal women.
\end{abstract}

Key words Self-assessment, Health status, Menopause, Family Health Strategy
Resumo O objetivo do estudo foi investigar a prevalência e os fatores associados à autopercepção negativa de saúde em mulheres climatéricas cadastradas na Estratégia Saúde da Família em um centro urbano brasileiro. Pesquisa transversal, com amostra aleatória de mulheres climatéricas. Foi utilizado um instrumento validado abordando dados sociodemográficos, comportamentais, relacionados ao estado e à autopercepção de saúde. A associação entre as variáveis estudadas e à autopercepção negativa de saúde foi verificada por análise bivariada seguida de regressão de Poisson, com variância robusta, em modelo hierarquizado. A prevalência de autopercepção negativa de saúde na população estudada foi de 41,6\%, em $761 \mathrm{mu}$ lheres. Entre as mulheres de 52 a 65 anos, 49,2\% apresentaram autopercepção negativa de saúde. Idade correspondente à pós-menopausa, escolaridade até oito anos de estudo, ter um companheiro, não ter um trabalho formal, uso atual do tabaco e sedentarismo foram associados à autopercepção negativa de saúde. A presença de sintomas climatéricos, sobrepeso e obesidade, o uso atual de medicamentos e a presença de doenças crônicas também se mostraram associados no modelo final. As associações observadas apontam para a necessidade de ações de promoção de saúde voltadas às mulheres climatéricas.

Palavras-chave Autoavaliação, Estado de saúde, Climatério, Estratégia Saúde da Família 


\section{Introdução}

O climatério representa um período de transição entre as fases reprodutiva e não reprodutiva da mulher. É um fenômeno endócrino, caracterizado pelo hipoestrogenismo progressivo, em decorrência do esgotamento dos folículos ovarianos, ocorrendo nas mulheres de meia idade ${ }^{1}$. A menopausa, um marco do período climatérico, é definida como a última menstruação, identificada retrospectivamente após 12 meses de amenorreia ${ }^{2}$. A idade média de sua ocorrência tem se mantido praticamente inalterada ao longo dos anos, ou seja, por volta dos 50 anos de idade ${ }^{3}$. Considerando a expectativa de vida para as mulheres em grande parte do mundo, é natural concluir que as mulheres passam cerca de um terço de suas vidas em um estado de carência hormonal.

Durante o climatério as mulheres passam por mudanças físicas, hormonais e psicossociais simultâneas. Fisicamente, existe uma tendência ao declínio no estado de saúde em decorrência do aparecimento das doenças crônicas associadas ao envelhecimento ${ }^{3}$. As mudanças hormonais no climatério estão relacionadas ao declínio da função folicular ovariana com flutuações hormonais e consequente instabilidade vasomotora. Como consequência dessas mudanças ocorrem sintomas como ondas de calor, ressecamento da mucosa vaginal e distúrbios do sono ${ }^{4}$. Sob o aspecto psicossocial, o climatério corresponde a uma série de mudanças na vida da mulher ${ }^{5}$. Acrescenta-se a esse conjunto de alterações o fato de que nesse período a composição familiar poder sofrer mudanças devido a morte ou divórcio, filhos podem deixar ou retornar ao lar, os pais tornaremse mais dependentes, agravando ainda mais o contexto das alterações orgânicas.

Avaliar a saúde da mulher climatérica e conhecer como ela própria percebe suas condições de saúde são medidas fundamentais para adoção de estratégias preventivas e de promoção de saúde, evitando morbidades e permitindo uma melhor qualidade nessa fase da vida das mulheres. Embora existam vários estudos acerca das condições clínicas associadas ao climatério, poucas pesquisas se dedicam à avaliação da autopercepção de saúde das mulheres durante esse período ${ }^{6}$.

A autopercepção de saúde é um indicador utilizado de forma crescente em estudos epidemiológicos tendo em vista sua validade e confiabilidade, associando-se fortemente com o estado real ou objetivo de saúde das pessoas, incorporando seus aspectos físicos, cognitivos e emocionais ${ }^{7,8}$. É uma variável simples de ser obtida e for- nece informações importantes acerca da população estudada por ser influenciada não apenas pela presença de doenças, mas também pelo bem -estar, nível de satisfação com a vida, capacidade funcional e qualidade de vida das pessoas ${ }^{9-13}$.

Estudos demonstram que a percepção de saúde feita pelo próprio indivíduo é um forte preditor de morbidade e mortalidade, mesmo se outros fatores como variáveis físicas, psicossociais e sociodemográficas forem controlados ${ }^{6,14}$. Alguns autores têm mostrado também que a autopercepção de saúde é uma medida de saúde que se associa com a utilização de serviços de saúde ${ }^{9}$. Por essas razões a autopercepção de saúde é considerada um indicador útil e uma ferramenta prática em inquéritos de saúde populacionais.

$\mathrm{O}$ presente estudo tem como objetivo investigar a prevalência e os fatores associados à autopercepção negativa de saúde das mulheres climatéricas cadastradas na Estratégia Saúde da Família (ESF) de um município norte mineiro.

\section{Métodos}

Foi realizado um estudo transversal, cuja população alvo foi composta pelas mulheres com idade entre 40 e 65 anos cadastradas nas Unidades Básicas de Saúde (UBS) da Estratégia Saúde da Família (ESF) na zona urbana de um grande centro do norte de Minas Gerais. A região representa uma área de transição entre o Sudeste, mais desenvolvido, e o Nordeste, menos desenvolvido, e caracteriza-se por seus contrastes socioeconômicos, sendo considerada uma área emblemática dos desafios do País.

A amostragem realizada foi do tipo probabilístico. As participantes foram selecionadas mediante sorteio, seguindo um plano amostral em dois estágios. Inicialmente, foram selecionados os conglomerados, representados pelas UBS, por amostragem aleatória simples. Das 73 unidades da ESF do município, 20 foram sorteadas para participar da amostra. Num segundo estágio, foram selecionadas as participantes do estudo de forma aleatória simples, com estratificação de acordo com o período do climatério. Foram consideradas na pré-menopausa as mulheres com idade entre 40 e 45 anos, na perimenopausa aquelas que tinham entre 46 e 51 anos e, na pósmenopausa, as que tinham entre 52 e 65 anos de idade $^{15}$. Na determinação do cálculo amostral, tomou-se como parâmetro o número de mulheres cadastradas nas equipes de saúde da ESF e uma frequência esperada de 50\% do evento (au- 
topercepção negativa de saúde), considerando a inexistência de dados prévios sobre o indicador e o fato dessa prevalência gerar o maior número amostral. O erro amostral admitido foi de 5\% e o nível de confiança de 95\%. O valor final foi multiplicado por um fator de correção para efeito do desenho (deff) igual a 2, obtendo-se, assim, um número mínimo de 760 mulheres a serem avaliadas.

Após o treinamento dos entrevistadores e antes do período da coleta de dados, conduziu-se um estudo piloto em uma unidade da ESF, com mulheres pertencentes ao grupo etário estudado e que não fizeram parte da amostra final. O estudo piloto permitiu que fossem testados na prática o questionário e o desempenho dos entrevistadores. Após essa fase, a pesquisa de campo foi iniciada. Ajustes no instrumento de coleta de dados não foram necessários.

Para coleta dos dados foi utilizado um questionário com questões obtidas de outros instrumentos validados ${ }^{16-18}$ referentes a aspectos sociodemográficos, comportamentais e relacionados ao estado de saúde. A variável dependente foi representada pela autopercepção de saúde e foi obtida por meio da pergunta: Em comparação com pessoas da sua idade, como você considera o seu estado de saúde?. As quatro categorias de resposta foram dicotomizadas em positiva (para as opções "muito bom" e "bom") e negativa (para as opções "regular” e "ruim").

As variáveis independentes foram subdividas em sociodemográficas, comportamentais e relacionadas ao estado de saúde. As variáveis sociodemográficas pesquisadas foram idade, cor da pele, escolaridade, estado civil e trabalho formal. As comportamentais foram representadas pela prática de atividade física, tabagismo atual e abuso do álcool. A atividade física foi verificada pelo Questionário Internacional de Atividade Física - IPAQ, versão curta ${ }^{16}$. Foram consideradas ativas aquelas mulheres que cumpriram as recomendações de atividade vigorosa: $\geq 3$ dias na semana e $\geq 20$ minutos por sessão; ou atividade moderada ou caminhada: $\geq 5$ dias na semana e $\geq 30$ minutos por sessão; ou qualquer atividade somada: $\geq 5$ dias na semana e $\geq 150$ minutos na semana. As sedentárias foram aquelas que não realizaram nenhuma atividade física por pelo menos 10 minutos contínuos durante a semana. As mulheres consideradas irregularmente ativas foram aquelas que realizaram atividade física, porém insuficientemente para serem classificadas como ativas, pois não cumpriram as recomendações quanto à frequência ou duração. $\mathrm{O}$ abuso de álcool, foi considerado o consumo de 4 ou mais doses de bebida alcoólica (sendo 1 dose igual a 1 lata de cerveja, 1 taça de vinho ou 1 dose de destilados como cachaça ou uísque) em uma única ocasião, nos últimos 30 dias ${ }^{17}$. Em relação ao estado de saúde, foram avaliados o uso atual de algum medicamento, a presença de morbidades autorrelatadas (hipertensão arterial, doenças do coração, colesterol elevado, diabetes, problema de coluna, depressão), menstruação nos últimos 12 meses, sintomas climatéricos de acordo com o índice de Kupperman ${ }^{18}$ e o cálculo do índice de massa corporal (IMC) utilizando-se as medidas antropométricas de massa corporal $(\mathrm{kg})$ e a estatura $(\mathrm{m})$ das mulheres avaliadas (para mensuração, utilizaram-se balanças da marca Filizola com precisão de $100 \mathrm{~g}$ e estadiômetro acoplado).

Para análise dos dados, utilizou-se o programa estatístico SPSS (Statistical Package for the Social Sciences), versão 21 . Inicialmente, foi realizada uma análise descritiva exploratória dos dados, com distribuição de frequências das variáveis do estudo. Em seguida foram realizadas análises bivariadas, buscando-se associações entre as variáveis independentes e a autopercepção negativa da saúde, com uso do teste qui-quadrado, sendo selecionadas para a análise multivariada, as variáveis associadas até o nível de 20\% ( $\mathrm{p} \leq 0,20)$.

$\mathrm{Na}$ fase analítica ajustada, foi utilizada a regressão de Poisson, com variância robusta, baseada em um modelo hierárquico em que o bloco das variáveis sociodemográficas foi considerado o determinante distal (primeiro nível). As variáveis relacionadas aos hábitos de vida compuseram o bloco seguinte (segundo nível) e as variáveis relacionadas ao estado de saúde como morbidades autorreferidas, uso de medicamentos, sintomas climatéricos e IMC compuseram o terceiro e último nível, mais proximal à variável dependente. Foram obtidas as razões de prevalências (RP) e seus respectivos intervalos de confiança de 95\% (IC 95\%), sendo adotado para o modelo final o nível de significância de 5\% ( $p<0,05)$. Todos os cálculos durante as análises foram realizados considerando a ponderação amostral (complex sample analysis).

Os participantes do estudo concordaram em participar da presente pesquisa de forma voluntária e assinaram o Termo de Consentimento Livre e Esclarecido, contendo o objetivo do estudo, procedimento de avaliação e caráter de voluntariedade da participação. O projeto do estudo foi previamente avaliado e aprovado pelo Comitê de Ética em Pesquisa das Faculdades Integradas Pitágoras de Montes Claros. 


\section{Resultados}

Foram entrevistadas 761 mulheres com idade entre 40 e 65 anos, sendo que dessas, $43,9 \%$ compreendiam o grupo etário correspondente à pósmenopausa (52 a 65 anos) e $56,1 \%$ os grupos pré e perimenopausa (40 a 51 anos). Mais da metade das mulheres referiu a cor da pele como parda $(65,6 \%)$, era casada $(64,4 \%)$ e tinha até 8 anos de estudo $(67,2 \%)$. Em relação aos hábitos de vida, maioria delas não fumava $(89,4 \%)$ e era irregularmente ativa (56,8\%). A Tabela 1 apresenta uma descrição detalhada em relação ao perfil sociodemográfico e comportamental da amostra.

Tabela 1. Características sociodemográficas e comportamentais de mulheres climatéricas, 2014.

\begin{tabular}{lrrr}
\hline \multicolumn{1}{c}{ Variáveis } & (n) & $\%$ & $\%^{*}$ \\
\hline Idade & & & \\
40 a 45 anos (pré-menopausa) & 224 & 28,4 & 29,4 \\
46 a 51 anos (perimenopausa) & 203 & 27,3 & 26,7 \\
52 a 65 anos (pós-menopausa) & 334 & 44,3 & 43,9 \\
Cor da pele & & & \\
Branca & 129 & 18,0 & 17,0 \\
Parda & 499 & 64,8 & 65,6 \\
Preta & 90 & 12,0 & 11,8 \\
Outras & 43 & 5,2 & 5,6 \\
Estado Civil & & & \\
Solteira & 71 & 9,3 & 9,3 \\
Casada & 490 & 65,3 & 64,4 \\
Separada/Divorciada & 123 & 15,4 & 16,2 \\
Viúva & 77 & 10,0 & 10,1 \\
Escolaridade & & & \\
Até 4 anos de estudo & 308 & 39,6 & 40,5 \\
5 a 8 anos de estudo & 203 & 26,8 & 26,7 \\
Mais de 8 anos de estudo & 250 & 33,6 & 32,8 \\
Trabalho formal & & & \\
Não & 446 & 59,0 & 58,6 \\
Sim & 315 & 41,0 & 41,4 \\
Tabagismo atual & & & \\
Sim & 81 & 10,0 & 10,6 \\
Não & 680 & 90,0 & 89,4 \\
Abuso do álcool & & & \\
Sim & 56 & 7,4 & 7,8 \\
Não & 705 & 92,6 & 92,2 \\
Atividade física & & & \\
Sedentárias & 231 & 30,7 & 30,3 \\
\hline Prregularmente ativas & 532 & 56,1 & 56,8 \\
Prvuito ativas & & & 12,9 \\
\hline
\end{tabular}

${ }^{*}$ Prevalências ajustadas, segundo a ponderação amostral.
As características do estado de saúde estão descritas na Tabela 2. Observa-se que $58,4 \%$ das mulheres avaliadas relataram autopercepção de saúde muito boa ou boa e $41,6 \%$ relataram autopercepção regular ou ruim. Mais da metade delas usava algum tipo de medicamento $(63,8 \%)$ e apresentava excesso de peso $(74,2 \%)$. Entre os principais problemas de saúde referidos destacaram-se os problemas de coluna $(53,2 \%)$, a hipertensão arterial $(47,9 \%)$ e o colesterol elevado $(41,0 \%)$.

As Tabelas 3 e 4 apresentam os resultados das análises bivariadas entre autopercepção de saúde e as variáveis independentes. Após análise múltipla, permaneceram no modelo hierarquizado as variáveis idade, escolaridade, situação conjugal, a falta de trabalho formal, o tabagismo atual, o

Tabela 2. Características do estado de saúde e autopercepção de saúde de mulheres climatéricas, 2014.

\begin{tabular}{|c|c|c|c|}
\hline Variáveis & $(\mathbf{n})$ & $\%$ & $\%{ }^{*}$ \\
\hline \multicolumn{4}{|c|}{ Menstruou nos últimos 12 meses } \\
\hline Não & 406 & 53,4 & 53,4 \\
\hline Sim & 355 & 46,6 & 46,6 \\
\hline \multicolumn{4}{|c|}{ Uso atual de algum medicamento } \\
\hline Sim & 486 & 63,5 & 63,8 \\
\hline Não & 275 & 36,5 & 36,2 \\
\hline \multicolumn{4}{|c|}{ Morbidades autorreferidas $* *$} \\
\hline \multirow[t]{2}{*}{ Pressão alta } & 365 & 48,1 & \\
\hline & & & 47,9 \\
\hline Colesterol alto & 312 & 40,7 & 41,0 \\
\hline Problema de coração & 100 & 13,9 & 13,2 \\
\hline Diabetes & 117 & 15,0 & 15,4 \\
\hline Problema de coluna & 405 & 52,0 & 53,2 \\
\hline Depressão & 174 & 22,9 & 22,8 \\
\hline \multicolumn{4}{|l|}{ IMC } \\
\hline Adequado & 198 & 26,1 & 26,0 \\
\hline Sobrepeso & 289 & 38,4 & 38,0 \\
\hline Obesidade & 274 & 35,5 & 36,0 \\
\hline \multicolumn{4}{|l|}{ Sintomas climatéricos } \\
\hline Presentes & 292 & 39,0 & 38,4 \\
\hline Ausentes & 469 & 61,0 & 61,6 \\
\hline \multicolumn{4}{|l|}{ Autopercepção de saúde } \\
\hline Muito bom & 110 & 14,1 & 14,4 \\
\hline Bom & 335 & 43,2 & 44,0 \\
\hline Regular & 241 & 32,7 & 31,7 \\
\hline Ruim & 75 & 10,0 & 9,9 \\
\hline
\end{tabular}

${ }^{*}$ Prevalências ajustadas, segundo a ponderação amostral. $\left({ }^{* *}\right)$ A somatória é maior que $100 \%$ pois algumas mulheres relataram mais de uma morbidade. 
Tabela 3. Associação entre características sociodemográficas e comportamentais e autopercepção de saúde em mulheres climatéricas, 2014 (análise bivariada).

\begin{tabular}{|c|c|c|c|c|c|c|}
\hline \multirow{3}{*}{ Variáveis } & \multicolumn{6}{|c|}{ Autopercepção de saúde } \\
\hline & \multicolumn{2}{|c|}{ Negativa } & \multicolumn{2}{|c|}{ Positiva } & \multirow{2}{*}{ p-valor } & \multirow{2}{*}{$\operatorname{RP}(\operatorname{IC~} 95 \%)^{*}$} \\
\hline & $(\mathbf{n})$ & $\%$ & (n) & $\%$ & & \\
\hline Idade & & & & & $<0,001$ & \\
\hline 52 a 65 anos & 166 & 49,2 & 171 & 50,8 & & $1,37(1,33-1,41)$ \\
\hline 40 a 51 anos & 151 & 35,7 & 273 & 64,3 & & 1,00 \\
\hline Escolaridade & & & & & $<0,001$ & \\
\hline Até 8 anos & 244 & 48,3 & 261 & 51,7 & & $1,73(1,67-180)$ \\
\hline 9 ou mais anos & 71 & 27,8 & 185 & 72,2 & & 1,00 \\
\hline Cor da pele & & & & & 0,070 & \\
\hline Não Branca & 261 & 41,8 & 363 & 58,2 & & $1,03(0,99-1,07)$ \\
\hline Branca & 55 & 40,4 & 82 & 59,6 & & 1,00 \\
\hline Estado Civil & & & & & 0,010 & \\
\hline Com companheiro & 210 & 42,2 & 287 & 57,8 & & $1,04(1,01-1,07)$ \\
\hline Sem companheiro & 107 & 40,6 & 157 & 59,4 & & 1,00 \\
\hline Trabalho formal & & & & & $<0,001$ & \\
\hline Não & 208 & 46,3 & 241 & 53,7 & & $1,32(1,28-1,36)$ \\
\hline Sim & 109 & 35,0 & 203 & 65,0 & & 1,00 \\
\hline Tabagismo atual & & & & & $<0,001$ & \\
\hline Sim & 42 & 55,9 & 34 & 44,1 & & $1,40(1,34-1,45)$ \\
\hline Não & 273 & 39,9 & 412 & 60,1 & & 1,00 \\
\hline Abuso do álcool & & & & & 0,210 & \\
\hline Sim & 24 & 42,9 & 32 & 57,1 & & $1,03(0,98-1,09)$ \\
\hline Não & 342 & 48,5 & 363 & 51,5 & & 1,00 \\
\hline Atividade física & & & & & $<0,001$ & \\
\hline Sedentárias & 115 & 49,3 & 119 & 50,7 & & $1,28(1,25-1,32)$ \\
\hline Ativas & 202 & 38,3 & 325 & 61,7 & & 1,00 \\
\hline
\end{tabular}

(*) RP: Razão de Prevalência; IC95\%: Intervalo de Confiança de 95\%.

sedentarismo, o uso de medicamentos e o autorrelato de pressão alta, colesterol alto, problema de coração, diabetes, problema de coluna, depressão, sobrepeso/obesidade, segundo o IMC, além da presença de sintomas climatéricos (Tabela 5).

\section{Discussão}

O presente estudo revelou uma elevada prevalência de autopercepção negativa da saúde (saúde regular ou ruim) entre as mulheres climatéricas. Estudo populacional que avaliou a presença de multimorbidades em mulheres brasileiras com a mesma faixa etária também apresentou resultados similares para autoavaliação negativa de saúde $^{19}$. Embora com diferentes categorias de respostas para autopercepção de saúde, estudo realizado em Florianópolis ${ }^{9}$ com pessoas idosas de ambos os sexos também mostrou elevada prevalência para autopercepção negativa de saúde. A análise desses resultados aproxima os resultados de autopercepção de saúde de mulheres climatéricas daqueles encontrados com a população idosa.

Com relação à idade, observou-se que entre as mulheres na pós-menopausa (faixa etária acima dos 52 anos de idade) houve um aumento na prevalência de uma pior autopercepção de saúde em relação às mulheres climatéricas mais jovens. Uma piora na percepção do estado de saúde relacionada com o envelhecimento já foi demonstrada em outros estudos, embora a maioria desses tenha sido realizada com a população geral ${ }^{20-23}$. Sabe-se que, à medida em que se envelhece, há uma diminuição na condição das pessoas em realizar atividades cotidianas que pode ser causada, anteceder, ou ocorrer de forma independente do aparecimento de morbidades e isso causa impacto na percepção de saúde dos indíduos ${ }^{21}$.

A associação entre a autopercepção de saúde e escolaridade já foi demonstrada em diversos es- 
Tabela 4. Associação entre características do estado de saúde e autopercepção de saúde em mulheres climatéricas, 2014 (análise bivariada).

\begin{tabular}{|c|c|c|c|c|c|c|}
\hline \multirow{3}{*}{ Variáveis } & \multicolumn{6}{|c|}{ Autopercepção de saúde } \\
\hline & \multicolumn{2}{|c|}{ Negativa } & \multicolumn{2}{|c|}{ Positiva } & \multirow{2}{*}{ p-valor } & \multirow{2}{*}{ RP $(\text { IC 95\%) })^{*}$} \\
\hline & $(\mathbf{n})$ & $\%$ & $(\mathbf{n})$ & $\%$ & & \\
\hline Menstruou nos últimos $12 \mathrm{~m}$ & & & & & $<0,001$ & \\
\hline Não & 193 & 47,6 & 213 & 52,4 & & $1,36(1,32-1,41)$ \\
\hline Sim & 124 & 34,8 & 231 & 65,2 & & 1,00 \\
\hline Uso de algum medicamento & & & & & $<0,001$ & \\
\hline Sim & 238 & 49,2 & 245 & 50,8 & & $1,73(1,67-1,80)$ \\
\hline Não & 79 & 28,3 & 199 & 71,7 & & 1,00 \\
\hline Pressão alta & & & & & $<0,001$ & \\
\hline Sim & 199 & 54,5 & 167 & 45,5 & & $1,83(1,77-1,88)$ \\
\hline Não & 117 & 29,7 & 278 & 70,3 & & 1,00 \\
\hline Colesterol alto & & & & & $<0,001$ & \\
\hline Sim & 167 & 54,0 & 143 & 46,0 & & $1,64(1,59-1,68)$ \\
\hline Não & 148 & 32,9 & 303 & 67,1 & & 1,00 \\
\hline Problema de coração & & & & & $<0,001$ & \\
\hline Sim & 68 & 64,6 & 38 & 35,4 & & $1,69(1,64-1,74)$ \\
\hline Não & 250 & 38,1 & 405 & 61,9 & & 1,00 \\
\hline Diabetes & & & & & $<0,001$ & \\
\hline Sim & 72 & 63,0 & 42 & 37,0 & & $1,67(1,62-1,72)$ \\
\hline Não & 244 & 37,7 & 403 & 62,3 & & 1,00 \\
\hline Problema de coluna & & & & & $<0,001$ & \\
\hline Sim & 214 & 54,0 & 182 & 46,0 & & $1,96(1,90-2,03)$ \\
\hline Não & 100 & 27,5 & 265 & 72,5 & & 1,00 \\
\hline Depressão & & & & & $<0,001$ & \\
\hline Sim & 100 & 57,4 & 74 & 42,6 & & $1,55(1,51-1,60)$ \\
\hline Não & 217 & 36,9 & 370 & 63,1 & & 1,00 \\
\hline IMC & & & & & $<0,001$ & \\
\hline Sobrepeso/obesidade & 253 & 45,0 & 309 & 55,0 & & $1,41(1,35-1,46)$ \\
\hline Adequado & 63 & 31,9 & 136 & 68,1 & & 1,00 \\
\hline Sintomas climatéricos & & & & & $<0,001$ & \\
\hline Presentes & 177 & 59,5 & 120 & 40,5 & & $1,95(1,89-2,00)$ \\
\hline Ausentes & 142 & 30,5 & 322 & 69,5 & & 1,00 \\
\hline
\end{tabular}

(*) RP: Razão de Prevalência; IC95\%: Intervalo de Confiança de 95\%.

tudos $^{24-27}$. No presente estudo observou-se que a escolaridade de até oito anos de estudo foi associada à autopercepção negativa de saúde. É possível que a baixa escolaridade comprometa a participação social da mulher em atividades que possam favorecer seu estado de saúde, como acesso à informação, aos cuidados de saúde em geral e às oportunidades sociais ao longo da vida ${ }^{28}$. Segundo dados do Vigitel, inquérito nacional realizado em todas as capitais brasileiras e no Distrito Federal, houve expressiva redução na frequência de autoavaliação negativa da saúde com aumento da escolaridade ${ }^{29}$.

Em relação ao arranjo familiar, as mulheres que relataram ter um companheiro avaliaram sua saúde de forma negativa, em relação àquelas sem companheiro. Este estudo apresenta dados semelhantes aos de outro estudo realizado com idosos, em que aqueles que moravam sozinhos avaliaram sua saúde de forma mais positiva em relação aos que moravam acompanhados ${ }^{28}$. Essa associação pode ser atribuída ao fato de que aquelas pessoas que vivem sozinhas apresentam melhores condições de saúde física, não dependendo de terceiros para o autocuidado.

A percepção negativa do estado de saúde esteve associada também à ausência de um trabalho formal, fato já constatado anteriormen$\mathrm{te}^{21}$. Essa associação ainda é ambígua quando se avaliam outros estudos. A partir de dados da 
Tabela 5. Associação entre características sociodemográficas, comportamentais e relacionadas à saúde e autopercepção negativa de saúde de mulheres climatéricas, 2014 (análise múltipla).

\begin{tabular}{lccc}
\hline \multicolumn{1}{c}{ Variáveis } & p-valor & $\mathbf{R P}^{*}$ & ${\text { IC } \mathbf{9 5} \mathbf{\%}^{* *}}^{*}$ \\
\hline Compontente Distal & $<0,001$ & 1,43 & $1,36-1,52$ \\
Idade $>52$ anos & $<0,001$ & 2,14 & $2,02-2,27$ \\
Escolaridade $<$ anos de estudo & $<0,001$ & 1,15 & $1,09-1,22$ \\
Com companheiro & $<0,001$ & 1,25 & $1,19-1,33$ \\
Sem trabalho formal & & & \\
Componente Intermediário & $<0,001$ & 1,77 & $1,64-1,93$ \\
Tabagismo atual & $<0,001$ & 1,36 & $1,29-1,44$ \\
Sedentárias & & & \\
Componente Proximal & 0,008 & 1,11 & $1,03-1,20$ \\
Uso de medicamentos atual & $<0,001$ & 1,55 & $1,44-1,66$ \\
Pressão alta & $<0,001$ & 1,39 & $1,31-1,48$ \\
Colesterol alto & $<0,001$ & 1,83 & $1,68-1,99$ \\
Problema de coração & $<0,001$ & 1,68 & $1,55-1,83$ \\
Diabetes & $<0,001$ & 2,38 & $2,24-2,52$ \\
Problema de coluna & $<0,001$ & 1,40 & $1,30-1,50$ \\
Depressão & $<0,001$ & 1,41 & $1,31-1,51$ \\
Sobrepeso/obesidade & $<0,001$ & 2,41 & $2,28-2,56$ \\
Sintomas climatéricos & & & \\
\hline
\end{tabular}

(*) RP: Razão de Prevalência. (**) IC95\%: Intervalo de Cofiança de 95\%.

Pesquisa Mundial de Saúde conduzida no Brasil, por exemplo, verificou-se que apenas entre os homens o desemprego esteve associado a uma autopercepção negativa da saúde, não sendo observada essa associação com as mulheres naquela ocasiã $0^{25}$. A progressiva inserção da mulher no mercado de trabalho e, ao mesmo tempo, algum tipo de sofrimento por não poder trabalhar ou a própria condição de desemprego podem associar-se, hipoteticamente, à autopercepção negativa de saúde.

Com relação aos hábitos de vida, verificou-se no presente estudo que o sedentarismo e o consumo atual de tabaco estiveram associados a uma autoavaliação negativa do estado de saúde. Já foi demonstrado que a prática regular de atividade física promove uma melhoria na qualidade de vida, através da manutenção da independência e autonomia das pessoas e, consequentemente, o bem-estar biopsicossocial'. O tabagismo é um reconhecido fator de risco para várias doenças e já foi também apontado como variável associada à percepção negativa da saúde ${ }^{24}$.

Os resultados deste estudo indicam que a presença de doenças crônicas entre as mulheres no climatério associa-se de maneira significativa com uma percepção negativa do estado de saúde. A presença de doenças crônicas implica em uma maior probabilidade de se avaliar a saúde de forma negativa ${ }^{19,20,24,26}$. Essa associação negativa é esperada, sobretudo, entre os idosos, podendo ser resultado de uma percepção de que ter saúde signifique ausência de doenças. Outro fator que também pode explicar a autopercepção negativa de saúde ligada à presença de comorbidades é o fato de que elas podem trazer limitações para atividades cotidianas e também para o autocuidado. Em estudo realizado na cidade de Belo Horizonte, com mulheres climatéricas com 11 ou mais anos de escolaridade, foi evidenciada associação entre autopercepção de saúde péssima/ruim e presença de depressão ${ }^{30}$. Esse dado pode sinalizar que a percepção do estado de saúde para essa população específica tenha comportamento similar às demais populações em que o indicador já foi estudado.

Além da presença de doenças crônicas, verificou-se neste estudo que o uso de medicamentos também esteve associado a uma pior percepção do estado de saúde. É esperado que o consumo regular de medicamentos implique, de fato, em uma sensação de fragilidade das condições de saúde ${ }^{9,10}$. O sobrepeso e a obesidade também foram associados a uma pior percepção do estado de saúde, fato já demonstrado em estudo prévio realizado com mulheres brasileiras com mais de 
$50 \operatorname{anos}^{31}$. Embora a obesidade esteja reconhecidamente relacionada com a presença de doenças crônicas como as doenças cardiovasculares, a associação entre IMC e autopercepção negativa de saúde persiste mesmo após o ajuste para a presença dessas morbidades.

Associação entre sintomas climatérios e autopercepção negativa da saúde foi verificada neste estudo. Esse achado alerta para a necessidade de maior valorização desses sintomas na abordagem à mulher na faixa etária de 40 a 65 anos. Já foi demonstrado que a maior intensidade de sintomas climatéricos associa-se à autopercepção negativa de saúde, sendo possível que essa associação seja causada pelo efeito negativo que os sintomas trazem ao estado psicológico da mulher ${ }^{32}$.

O presente estudo apresenta algumas limitações que precisam ser consideradas no processo de interpretação e generalização dos resultados. A população estudada compreendeu apenas mulheres cadastradas nas equipes do sistema público de saúde, da ESF. Esse público é, em sua maioria, de classe socioeconômica mais baixa e, portanto, os resultados não podem ser extrapolados para a população geral. O delineamento transversal não permite que se façam inferências de causa-efeito em relação à autopercepção negativa de saúde e as variáveis estudadas. Outra possível limitação é que o uso de variáveis autorreferidas pode implicar em informações incorretas, embora treinamento da equipe de campo e realização de estudo piloto tenham sido realizados para minimizar possíveis erros no momento da coleta.

Por outro lado, destaca-se a relevância do presente estudo cujos resultados apresentados representam informações importantes sobre a saúde de uma parcela quase sempre negligenciada da população brasileira, as mulheres climatéricas. A análise de uma amostra representativa das mulheres climatéricas de um grande centro possibilitou a identificação de fatores associados à autopercepção negativa de saúde. Os resultados apresentados contribuem para que outras pesquisas envolvendo aspectos de promoção e autopercepção de saúde sejam desenvolvidas, com objetivo de melhorar a qualidade de vida das mulheres, em especial ao atingir a fase do climatério.

\section{Colaboradores}

VH Silva, JSB Rocha e AP Caldeira participaram igualmente de todas as etapas de elaboração do artigo. 


\section{Referências}

1. Malheiros ESA, Chen MBC, Silva DSM, DIAS CLL, Brito LGO, Pinto-Neto AM, Brito LMO. Síndrome climatérica em uma cidade do Nordeste brasileiro: um inquérito domiciliar. Rev Bras Ginecol Obstet 2014; 36(4):163-169.

2. World Health Organization (WHO). Report of a WHO Scientifc Group: Research on the Menopause in the 1990's. Geneva: WHO; 1996. [WHO Technical Report Series 866].

3. Brasil. Ministério da Saúde (MS). Manual de Atenção à Mulher no Climatério/Menopausa. Brasília: Editora do Ministério da Saúde; 2008.

4. Pedro AO, Pinto-Neto AA, Costa-Paiva LHS, Osis MJD, Hardy EE. Síndrome do climatério: inquérito populacional domiciliar em Campinas, SP. Rev Saude Publica 2003; 37(6):735-742.

5. Silveira IL, Petronilo PA, Souza MO, Nogueira TD, Silva C, Duarte JMBP, Maranhão TMO, Azevedo GD. Prevalência de sintomas do climatério em mulheres dos meios rural e urbano no Rio Grande do Norte, Brasil. Rev Bras Ginecol Obstet 2007; 29(8):420-427.

6. DeSalvo KB, Bloser N, Reynolds K, He J, Muntner P. Mortality Prediction with a Single General Self-Rated Health Question A Meta-Analysis. J Gen Intern Med 2005; 20(3):267-275.

7. Freidoony L, Chhabi R, Kim CS, Park MB, Kim CB. The Components of Self-Perceived Health in the Kailali District of Nepal: A Cross-Sectional Survey. Int J Environ Res Public Health 2015; 12(3):3215-3231.

8. Perrucio AV, Katz JN, Losina E. Health burden in chronic disease: Multimorbidity is associated with self-rated health more than medical comorbidity alone. J Clin Epidemiol 2012; 65(1):100-106.

9. Confortin SC, Giehl MWC, Antes DL, Schneider IJC, d'Orsi E. Autopercepção positiva de saúde em idosos: estudo populacional no Sul do Brasil. Cad Saude Publica 2015; 31(5):1049-1060.

10. Pagotto V, Bachion MM, Silveira EA. Autoavaliação da saúde por idosos brasileiros: revisão sistemática da literatura. Rev Panam Salud Publica 2013; 33(4):302-310.

11. Pagotto V, Nakatani A, Silveira E. Fatores associados à autoavaliação de saúde ruim em idosos usuários do Sistema Único de Saúde. Cad Saude Publica 2011; 27(8):1593-1602.

12. Kochergin CN, Proietti FA, César CC. Comunidades quilombolas de Vitória da Conquista, Bahia, Brasil: autoavaliação de saúde e fatores associados. Cad Saude Publica 2014; 30(7):1487-1501.

13. Reichert FF, Loch MR, Capilheira MF. Autopercepção de saúde em adolescentes, adultos e idosos. Cien Saude Colet 2012; 17(12):3353-3362.

14. Eriksson, Undén AL, Elofsson S. Self-rated health. Comparisons between three different measures. Results from a population study. Int J Epidemiol 2001; 30(2):326-333.

15. Fernandes CE, Stephan C, Nasser EJ, Ferreira JAS, Melo NR, Peixoto S. Síndrome do Climatério. Rev Bras Med 2005; 62(12):1-9.
16. Craig CL, Marshall AL, Sjostro M, Bauman AE, Booth ML, Ainsworth BE, Pratt M, Ekelund U, Yngve A, Sallis JF, Oja P. International Physical Activity Questionnaire: 12-Country Reliability and Validity. Med Sci Sports Exerc 2003; 35(8):1381-1395.

17. Machado IE, Lana FCF, Felisbino-Mendes MS, Malta DC. Factors associated with alcohol intake and alcohol abuse among women in Belo Horizonte, Minas Gerais State, Brazil. Cad Saude Publica 2013; 29(7):1449-1459.

18. Kupperman HS, Blatt MHG. Menopausal indice. J Clin Endocrinol 1953; 13(1):688-694.

19. Machado VSS, Valadares A, Costa-Paiva LS, Moraes SS, Pinto-Neto AM. Multimorbidity and associated factors in Brazilian women aged 40 to 65 years: a population-based study. Menopause 2012; 19(5):569-575.

20. Alves LS, Rodrigues RN. Determinantes da autopercepção de saúde entre idosos do Município de São Paulo, Brasil. Rev Panam Salud Publica 2005; 17(5/6):333-341.

21. Traebert J, Bortoluzzi MC, Kehrig RT. Autopercepção das condições de saúde da população adulta, sul do Brasil. Rev Saude Publica 2011; 45(4):789-793.

22. Agostinho MR, Oliveira MC, Pinto MEB, Balardin GU, Harzheim E. Autopercepção da saúde entre usuários da Atenção Primária em Porto Alegre, RS. Rev Bras Med. Fam. e Comum 2010; 5(17):9-15.

23. Barros MBA, Zanchetta LM, Moura EC, Malta DC. Auto-avaliação de saúde e fatores associados, Brasil 2006. Rev Saude Publica 2009; 43(2):27-37.

24. Pavão ALB, Werneck GL, Campos MR. Autoavaliação do estado de saúde e a associação com fatores sociodemográficos, hábitos de vida e morbidade na população: um inquérito nacional. Cad Saude Publica 2013; 29(4):723-734.

25. Szwarcwald CL, Souza-Junior PRB, Esteves MAP, Damacena GN, Viacava F. Socio-demographic determinants of self-rated health in Brazil. Cad Saude Publica 2005; 21(Supl.):S54-S64.

26. Oliveira SKM, Pereira MM, Guimarães LS, Caldeira AP. Autopercepção de saúde em quilombolas do norte de Minas Gerais, Brasil. Cien Saude Colet 2015; 20(9):2879-2890.

27. Borim FSA, Neri AL, Francisco PMSB, Barros MBA. Dimensões da autoavaliação de saúde em idosos. Rev Saude Publica 2014; 48(5):714-722.

28. Borim FSA, Barros MBA, Neri AL. Autoavaliação da saúde em idosos: pesquisa de base populacional no Município de Campinas, São Paulo, Brasil. Cad Saude Publica 2012; 28(4):769-780.

29. Brasil. Ministério da Saúde (MS). Vigitel Brasil 2014: vigilância de fatores de risco e proteção para doenças crônicas por inquérito telefônico. Brasília: MS; 2015.

30. Machado VSS, Valadares ALR, Costa-Paiva L, Morais SS, Pinto-Neto AM. Morbidades e fatores associados em mulheres climatéricas: estudo de base populacional em mulheres com 11 anos ou mais de escolaridade. Rev Bras Ginecol Obstet 2012; 34(5):215-220. 
31. Machado VSS, Valadares ALR, Costa-Paiva LH, Osis MJ, Sousa MH, Pinto-Neto AM. Factors associated with the self-perception of health among Brazilian women 50 years or older: a population-based study. Menopause 2013; 20(10):1055-1060.

32. Lui Filho JF, Baccaro LFC, Fernandes T, Conde DM, Costa-Paiva L, Pinto-Neto AM. Epidemiologia da menopausa e dos sintomas climatéricos em mulheres de uma região metropolitana no sudeste do Brasil: inquérito populacional domiciliar. Rev Bras Ginecol Obstet 2015; 37(4):152-158.

Artigo apresentado em 19/04/2016

Aprovado em 21/08/2016

Versão final apresentado em 23/08/2016 